\title{
Quantum Corrections to Semiclassical Transport in Nanoscale Devices using Entropy Principles
}

\author{
J. P. Bourgade, P. Degond, N. Mauser*, and C. Ringhofer* \\ MIP, UMR 5640 Université Paul Sabatier 118, route de Narbonne, 31062 TOULOUSE, FRANCE, \\ email: bourgade@mip.ups-tlse.fr, degond@mip.ups-tlse.fr \\ * Wolfgang Pauli Institut, Universität Wien, A-1090 Vienna, Austria and \\ Department of Mathematics, Arizona State University, Tempe, Arizona 85287-1804, USA \\ email: Norbert.Mauser@univie.ac.at, ringhofer@asu.edu
}

\begin{abstract}
We derive a modification of the semiclassical Fermi Golden Rule collision operator based on quantum thermodynamic principles. The resulting operator is nonlocal in space and acknowledges the presence of steep potential gradients and potential barriers. The resulting quantum mechanical transport equation - the Wigner quantum Boltzmann equation - increases the corresponding quantum mechanical entropy and is therefore well posed.
\end{abstract}

\section{INTRODUCTION}

The simulation of nanoscale devices requires, at least rudimentarily, the inclusion of quantum effects. Quite a lot of attention has been paid to the quantum mechanical modeling of free (collisionless) transport. Efforts here include the direct solution of the Schrödinger equation [2], [11], macroscopic moment equations (quantum hydrodynamic models) [9], [10], and extensions of semiclassical Monte Carlo methods, either directly [13], [8] or via effective potential approaches [6], [1], [12]. Comparably little work has been done on the inclusion of quantum effects into collision operators. Collisions can be treated quite rigorously using Green's functions or by Wigner function methods for the phonon system [7]. Using classical collision operators in collusion with quantum corrected transport models has the disadvantage that equilibrium solutions of the resulting system do not correspond to admissible quantum mechanical states, since the semiclassical phonon collision operator increases the classical entropy and not the correct quantum mechanical entropy, given by $S=-\operatorname{Tr}(\rho \cdot \ln \rho)$, where $\rho$ denotes the density matrix of the mixed state. In this work we modify the semiclassical phonon collision operator, based on Fermi's Golden Rule, such that the resulting modification dissipates a quadratic approximation of the quantum mechanical entropy relative to the Hamiltoinian,

$S_{\text {rel }}=-\operatorname{Tr}[\rho \cdot(\ln \rho+H)]$ near thermal equilibrium. The final result is a quantum Boltzmann equation for the Wigner function $f_{w}$ of the form

$$
\partial_{t} f_{w}+\left[\varepsilon(\mathbf{k})+V(\mathbf{r}), f_{w}\right]_{q}=C_{q}\left[f_{w}\right]
$$

where $[*, *]_{q}$ denotes the usual quantum commutator in the Wigner picture. $C_{q}$ denotes the modified Fermi Golden Rule collision operator, which has the following properties:

- As in Fermi's Golden Rule, the change in energy, due to a collision event equals $\hbar \omega$, where $\omega$ denotes the dominant phonon frequency.

- $C_{q}$ increases the quadratic approximation of the relative quantum entropy $S_{r e l}$ around thermal equilibrium.

- $C_{q}$ is nonlocal in the spatial variable as well as in the wave vector. The spatial nonlocality becomes more pronounced for large electric fields.

The organization of this paper is the following. In Section II we define the quadratic approximation to the relative entropy functional $S_{\text {rel }}$ around thermal equilibrium and give a formula for its evaluation in terms of the eigenfunctions and eigenvalues of the Hamiltonian $H$. The entropic collision operator $C_{q}$ in (1) is derived in Section III and the resulting quantum Boltzmann equation is discussed in Section IV. Numerical results are presented in Section V. 


\section{THE ENTROPY CLOSE TO THERMAL EQUILIBRIUM}

Since the phonon collision operator models the interaction of electrons with a background of phonons, which is assumed to be in equilibrium, it should increase the entropy relative to the Hamiltonian. The relative entropy is given by $S_{\text {rel }}(\rho)=$ $-\operatorname{Tr}[\rho \cdot(\ln \rho+H)]$, where $H$ denotes the usual electron Hamiltonian $\varepsilon(\mathbf{k})+V(\mathbf{r})$. The present paper is concerned with a perturbation theory close to thermal equilibrium. That is, we replace $S_{r e l}$ by its quadratic approximation around the global maximum of $S_{\text {rel }}$, given by $\rho_{\text {equ }}=\exp (-\beta H)$, with $\beta$ the inverse ambient temperature. The quadratic approximation to the relative entropy near thermal equilibrium is then given by the quadratic form $S_{2}(\rho)=\frac{1}{2} D^{2} S_{\text {rel }}\left(\rho_{\text {equ }}\right)(\rho, \rho)$, where $D^{2} S_{\text {rel }}$ denotes the second Frechet derivative of $S_{\text {rel }}$. This quadratic entropy functional can be expressed in terms of a scalar product on the space of density matrices in the form $S_{2}(\rho)=-\frac{1}{2} \operatorname{Tr}\left[\rho \cdot u_{\rho}\right]$ where the relation between $\rho$ and $u_{\rho}$ is given by $\rho=$ $D \exp (-\beta H) u_{\rho}$, where again $D \exp$ denotes the Frechet derivative of the matrix exponential. Written in terms of the eigenbasis of the Hamiltonian, the relation between $\rho$ and $u_{\rho}$ is given by

$$
\begin{gathered}
\rho(x, y)=\sum_{k j} \psi_{k}(x) \rho_{k j} \psi_{j}(y), \\
u_{\rho}(x, y)=\sum_{k j} \psi_{k}(x) u_{k j} \psi_{j}(y), \\
\rho_{k j}=\frac{e^{-\beta \lambda_{j}}-e^{-\beta \lambda_{k}}}{\beta\left(\lambda_{k}-\lambda_{j}\right)} u_{k j} \\
S_{2}(\rho)=\sum_{k j}\left|u_{k j}\right|^{2} \frac{e^{-\beta \lambda_{j}}-e^{-\beta \lambda_{k}}}{\beta\left(\lambda_{k}-\lambda_{j}\right)}
\end{gathered}
$$

where $\psi_{k}$ and $\lambda_{j}$ denote the eigenfunctions and energies of the Hamiltonian $H$. The formula (2) can be derived from computing the derivative $D \exp$ of the matrix exponential by linearizing the corresponding Bloch equation [4], [5] and expressing the solution in terms of the eigenfunctions of the Hamiltonian. Note, that this represents the quantum mechanical equivalent of the classical entropy functional $S_{2}^{\text {class }}(f)=-\int d \mathbf{k} \int d \mathbf{r} e^{\beta H(\mathbf{k}, \mathbf{r})} f(\mathbf{k}, \mathbf{r})^{2}$, which is well known to be increased by the dynamics of the semiclassical Boltzmann equation.

\section{Fermi's GOLdEN RULE}

The electron - phonon interaction based on Fermi's Golden rule is given by the collision operator

$$
\begin{gathered}
C_{F G R}[f](\mathbf{k}, \mathbf{r}, t)=-\kappa(\mathbf{r}, \mathbf{k}) f(\mathbf{r}, \mathbf{k}, t)+ \\
\sum_{\nu= \pm 1} \int c_{\nu} \delta\left(\varepsilon(\mathbf{k})-\varepsilon\left(\mathbf{k}^{\prime}\right)+\nu \hbar \omega\right) f\left(\mathbf{k}^{\prime}, \mathbf{r}, t\right) d \mathbf{k}^{\prime}
\end{gathered}
$$

which states that in the course of the interaction with the lattice an amount $\hbar \omega$ of energy is either gained or lost by creation or annihilation of a phonon. At the same time, the probabilities $c_{1}, c_{-1}$ for the emission and absorption of a phonon in the infinitesimal time $d t$ always satisfy $\frac{c_{1}}{c_{-1}}=e^{\beta \hbar \omega}$ where $\beta$ is the inverse ambient temperature. For this reason, the collision operator $C_{F G R}$ can be expressed in weak form as

$$
\int \psi(\mathbf{k}) C_{F G R}[f](\mathbf{k}, \mathbf{r}) d \mathbf{k}=
$$

$-\frac{1}{2} \int\left[\psi(\mathbf{k})-\psi\left(\mathbf{k}^{\prime}\right)\right] K\left(\mathbf{k}, \mathbf{k}^{\prime}\right)\left[g(\mathbf{k}, \mathbf{r})-g\left(\mathbf{k}^{\prime}, \mathbf{r}\right)\right] d \mathbf{k k}^{\prime}$ where $\psi$ is an arbitrary test function, $g(\mathbf{k}, \mathbf{r})=$ $e^{\beta \varepsilon(\mathbf{k})} f(\mathbf{k}, \mathbf{r})$ holds, and the symmetrized kernel $K$ is given by

$$
\begin{gathered}
K\left(\mathbf{k}, \mathbf{k}^{\prime}\right)= \\
c_{0} \sum_{\nu= \pm 1} \delta\left(\varepsilon(\mathbf{k})-\varepsilon\left(\mathbf{k}^{\prime}\right)+\nu \hbar \omega\right) e^{-\frac{\beta}{2}\left(\varepsilon(\mathbf{k})+\varepsilon\left(\mathbf{k}^{\prime}\right)\right)}, \\
c_{0}=e^{-\frac{\beta \hbar \omega}{2}} c_{1}=e^{\frac{\beta \hbar \omega}{2}} c_{-1}
\end{gathered}
$$

From the form (5) it can be seen that the Fermi Golden Rule operator $C_{F G R}$ dissipates the classical entropy, given by the functional $S_{2}^{\text {class }}[f]=$ $-\int e^{\beta \varepsilon(\mathbf{k})} f(\mathbf{k}, \mathbf{r})^{2} d \mathbf{r k}$. By choosing the test function $\psi=g=e^{\beta \varepsilon(\mathbf{k})} f(\mathbf{k}, \mathbf{r})$ it is immediate that $\int g C_{F G R}[f] d \mathbf{k r} \leq 0$ holds and therefore the homogenous Boltzmann equation $\partial_{t} f=C_{F G R}[f]$ increases the classical entropy functional $S_{2}^{\text {class }}[f]$. The key point here is that the Fermi Golden Rule collision operator is completely determined by four properties, namely

1) A collision results in a gain or loss of an amount $\hbar \omega$ of energy.

2) Charge is conserved.

3) The operator increases the classical entropy. (The form of the entropy, i.e. $\beta$ governs the ration $\frac{c_{1}}{c_{-1}}$ in (5).) 
4) The scattering frequency, or the mean free path which determines the absolute size of $c_{ \pm 1}$, or alternatively, $c_{0}$ in (6).

The natural quantum thermodynamic modification of the classical Fermi Golden Rule operator consists therefore of replacing the classical entropy by the quantum entropy derived in Section II and keeping the other three points. This means, that the collision operator $C_{q}$ is given by (5) where the function $g_{w}$ is now computed from the Wigner function $f_{w}$ by $g_{w}=W\left[u_{\rho}\right], \rho=W^{-1}\left[f_{w}\right]$, where $W$ and $W^{-1}$ denote the usual Wigner - Weyl transform and its inverse. So, to evaluate $C_{q}\left[f_{w}\right]$, given the Wigner function $f_{w}$ the following steps have to be taken

1) Compute the representation of the density matrix $\rho$, corresponding to the Wigner function $f_{w}$, in the basis, given by the eigenfunctions of the Hamiltonian $H$.

2) Compute $u_{\rho}$ according to (2).

3) Compute the entropy variable $g_{w}$ in the Wigner picture as the Wigner - Weyl transform of $u_{\rho}$, i.e. $g_{w}=W\left[u_{\rho}\right]$.

4) Evaluate the Fermi Golden Rule collision operator (4) by replacing the classical density function $f$ by $e^{-\beta \varepsilon} g_{w}$.

The resulting collision operator expresses the same principle as Fermi's Golden Rule, namely the gain / loss of an amount $\hbar \omega$ of energy. However it increases the quantum entropy functional $S_{2}$ from Section II. Because of the properties of the Wigner transform, and the symmetry $S_{2}$ we have

$$
\partial_{t} S_{2}[\rho]=-\operatorname{Tr}\left[u_{\rho} \cdot \partial_{t} \rho\right]=-\int g \partial_{t} f_{w} d \mathbf{k r} \mathbf{r} .
$$

Therefore, multiplying the homogeneous equation $\partial_{t} f_{w}=C_{q}\left[f_{w}\right]$ by the entropy variable $g_{w}$ and integrating w.r.t. $\mathbf{k}$ and $\mathbf{r}$ yields $\partial_{t} S_{2} \geq 0$ because of the entropic weak form (5) of the classical Fermi Golden Rule operator $C_{F G R}$.

Remark: The modified collision operator $C_{q}$ is nonlocal in the spatial variable $\mathbf{r}$ because of the nonlocal relation between $f_{w}$ and $g_{w}$. This nonlocality becomes more pronounced in the presence of large electric fields, since in this case the support of the wave functions $\psi_{k}$ of the Hamiltonian are spread out over a larger region. In the classical limit ( $\hbar \rightarrow 0$ formally) the relation between the Wigner function $f_{w}$ and the entropy variable $g_{w}$ reduces to $f_{w}=e^{-\beta \varepsilon} g_{w}$ and the modified collision operator $C_{q}$ reduces to the classical Fermi Golden Rule.

\section{The QUANTUM BolTZMANN EQUATION}

The resulting transport equation in the Wigner picture is then of the form

$$
\partial_{t} f_{w}+\left[\varepsilon(\mathbf{k})+V(\mathbf{r}), f_{w}\right]_{q}=C_{q}\left[f_{w}\right],
$$

where $[*, *]_{q}$ denotes the usual quantum commutator in the Wigner picture. Using pseudo - differential operator notation, it is of the form

$$
\begin{gathered}
{\left[H, f_{w}\right]_{q}=} \\
\sum_{\nu= \pm 1} \frac{\nu}{i \hbar}\left[\varepsilon\left(\mathbf{k}+\frac{i \nu}{2} \nabla_{\mathbf{r}}\right)+V\left(\mathbf{r}-\frac{i \nu}{2} \nabla_{\mathbf{k}}\right)\right] f_{w}(\mathbf{k}, \mathbf{r}),
\end{gathered}
$$

where functions of gradients are defined in the usual way via Fourier transforms. Note, that the transport term only reduces to its classical equivalent $\frac{1}{\hbar} \nabla_{\mathbf{k}} \varepsilon \nabla_{\mathbf{r}} f_{w}$ in the case of quadratic $\varepsilon$, i.e. for parabolic band structures. By virtue of construction, the quadratic approximation $S_{2}$ of the entropy functional around thermal equilibrium is increased by the collision operator $C_{q}$. We now give a simple argument for the fact that the entropy is conserved by the quantum commutator $[*, *]_{q}$. Due to the cyclicity of the trace, we have that. $\operatorname{Tr}\left(a \cdot\left[H, e^{a}\right]_{q}\right)=0$ holds for any density matrix $a$ Differentiating this relation twice yields

$$
\begin{gathered}
\operatorname{Tr}\left(\delta a_{1} \cdot\left[H, e^{a}\right]_{q}+\delta a_{1} \cdot\left[H, D \exp (a) \delta a_{2}\right]_{q}+\right. \\
\left.\delta a_{2} \cdot\left[H, D \exp (a) \delta a_{1}\right]_{q}+a \cdot\left[H, D^{2} \exp (a)\left(\delta a_{1}, \delta a_{2}\right)\right]_{q}\right) \\
=0
\end{gathered}
$$

Setting $a=-H, \delta a_{1}=\delta a_{2}=u, D \exp (a) \delta a_{1}=$ $\rho$, and using the fact that a density matrix commutes with its exponential, yields $2 \operatorname{Tr}\left(u \cdot[H, \rho]_{q}\right)=0$. Thus, in the Wigner picture, where $u$ corresponds to $g_{w}$ and $\rho$ corresponds to $f_{w}$, we have

$$
\int g_{w}\left[H, f_{w}\right]_{q} d \mathbf{k} d \mathbf{r}=0
$$

which means that the quadratic entropy approximation $S_{2}$ is conserved by the quantum commutator $[*, *]_{q}$ as well. Integrating the quantum Boltzmann equation against the entropy variable $g_{w}$ yields $\partial_{t} S_{2}\left[f_{w}\right]=-\int g_{w} \partial_{t} f_{w} d \mathbf{k} d \mathbf{r} \geq 0$ and the total entropy is increased due to collisions. 


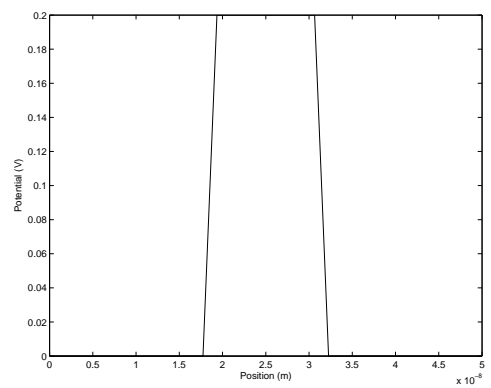

Fig. 1. Potential

\section{DISCUSSION AND PRELIMINARY SIMULATION RESULTS}

We have presented a relatively inexpensive extension of the semiclassical Fermi Golden rule collision operator. In fully quantum mechanical simulations, using the Wigner Boltzmann equation (7) this collision operator dissipates an approximation of the correct quantum entropy and the resulting problem is therefore well posed. We conclude by computing the steady states of the resulting collision operator, i.e. the equivalent of the Maxwellian, in the absence of the transport terms. In this case, the steady state entropy variable $g_{w}$ in Section II is given by a classical Maxwellian and the equilibrium density function $f_{w}$ is given by by the transformation outlined above. A simple barrier potential, shown in Figure 1, has been used. Figure 2 shows the steady state Wigner function corresponding to the classical global entropy maximum, i.e. for $g_{w}=e^{-\beta \varepsilon(\mathbf{k})-\beta V(\mathbf{r})}$. Figure 3 shows the Wigner function for a single particle inside the potential well. Note that the Wigner function in Figure 3 becomes locally negative, indicating that the integral kernel corresponding to the modified collision operator contains negative elements. Therefore, particle based discretizations of the Wigner - Boltzmann equation (7) will exhibit the same difficulties as particle discretizations of the collisionless Wigner equation (see [8]).

\section{ACKNOWLEDGEMENT}

This work was supported by NSF-ECS grant Nr. 021-8008, FWF START project Y-137-TEC and the Wolfgang Pauli Institute.

\section{REFERENCES}

[1] S. Ahmed, C. Ringhofer, D. Vasileska, Journal of Computational Electronics 2113 (2003).

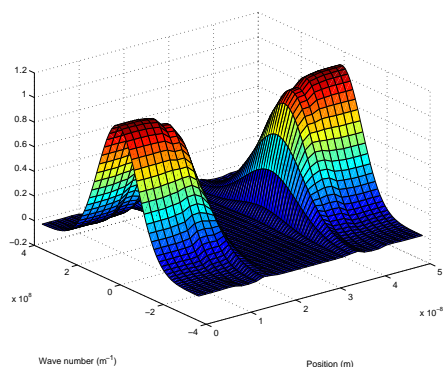

Fig. 2. Wigner function $f_{w}$ for a constant entropy variable $\phi$

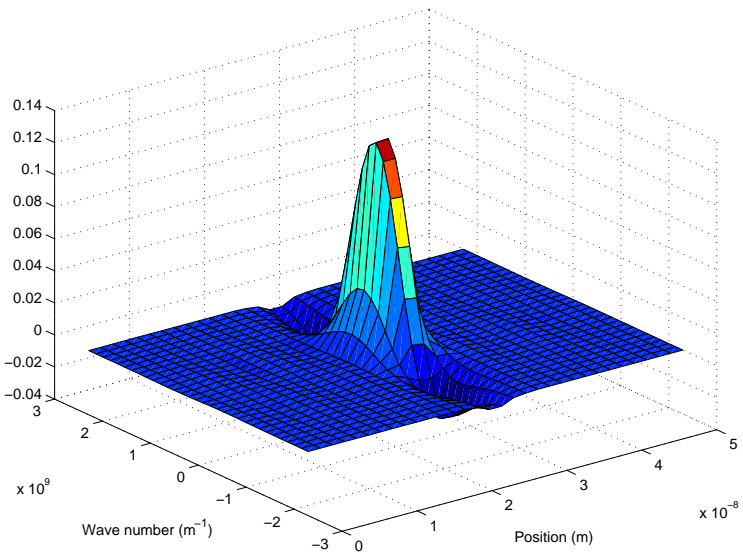

Fig. 3. Wigner function $f_{w}$ corresponding to $\phi$ in Figure ??

[2] N Ben Abdallah, Journal of Statistical Physics 90 3-4 (1998).

[3] J.-P. Bourgade, P. Degond, F. Mehats, C. Ringhofer On quantum extensions to classical Spherical Harmonics Expansion / Fokker-Planck models, to appear, Journal of Mathematical Physics (2006).

[4] P. Degond, C. Ringhofer, Journal Stat. Phys. 112 (3): 587-628 (2003).

[5] P. Degond, F. Mehats, C. Ringhofer, Journal Stat. Phys. 118 625-667 (2005).

[6] D. Ferry, H.Grubin, Solid State Physics 49:283 (1995).

[7] C. Jacoboni, A. Bertoni, P.Bordone, R. Brunetti, Math. Comp. Simul 5567 (2001).

[8] L. Shifren, C. Ringhofer, D.Ferry, IEEE Trans. Electron Devices 50 (3):769-773 (2002).

[9] C Gardner, C. Ringhofer, Phys. Rev E 58780 (1998).

[10] I. Gasser, A. Jüngel, Zeitschrift für Angewandte Mathematik und Physik 4845 (1997).

[11] C. Lent, D. Kirkner, J. Applied Physics 67 (10) 6353 (1990).

[12] C. Ringhofer, C. Gardner, D. Vasileska, Inter. J. on High Speed Electronics and Systems 13 771-803 (2003).

[13] R.Wyatt, Journal Chem. Phys. 1114406 (1999). 